\title{
Recombination from polar InGaN/GaN quantum well structures at high excitation carrier densities
}

DOI:

10.1103/PhysRevB.98.155301

\section{Document Version}

Accepted author manuscript

Link to publication record in Manchester Research Explorer

\section{Citation for published version (APA):}

Christian, G. M., Schulz, S., Kappers, M. J., Humphreys, C. J., Oliver, R. A., \& Dawson, P. (2018). Recombination from polar InGaN/GaN quantum well structures at high excitation carrier densities. Physical Review B, 98(15). https://doi.org/10.1103/PhysRevB.98.155301

\section{Published in:}

Physical Review B

\section{Citing this paper}

Please note that where the full-text provided on Manchester Research Explorer is the Author Accepted Manuscript or Proof version this may differ from the final Published version. If citing, it is advised that you check and use the publisher's definitive version.

\section{General rights}

Copyright and moral rights for the publications made accessible in the Research Explorer are retained by the authors and/or other copyright owners and it is a condition of accessing publications that users recognise and abide by the legal requirements associated with these rights.

\section{Takedown policy}

If you believe that this document breaches copyright please refer to the University of Manchester's Takedown Procedures [http://man.ac.uk/04Y6Bo] or contact uml.scholarlycommunications@manchester.ac.uk providing relevant details, so we can investigate your claim.

\section{OPEN ACCESS}




\title{
Recombination from polar InGaN/GaN quantum well structures at high excitation carrier densities
}

\author{
G. M. Christian, ${ }^{1, *}$ S. Schulz, ${ }^{2}$ M. J. Kappers, ${ }^{3}$ C. J. Humphreys,${ }^{3,4}$ R. A. Oliver, ${ }^{3}$ and P. Dawson ${ }^{1}$ \\ ${ }^{1}$ School of Physics and Astronomy, Photon Science Institute, \\ University of Manchester, Manchester, M13 9PL, UK \\ ${ }^{2}$ Photonics Theory Group, Tyndall National Institute, \\ University College Cork, Cork, T12 R5CP, Ireland \\ ${ }^{3}$ Department of Materials Science and Metallurgy, 27 Charles Babbage Road, \\ University of Cambridge, Cambridge, CB3 OFS, UK \\ ${ }^{4}$ School of Engineering and Materials Science, Queen Mary University of London, London, E1 4NS, UK
}

(Dated: October 2, 2018)

\begin{abstract}
In this paper we report on the emergence of a high energy band at high optically excited carrier densities in the low temperature photoluminescence spectra from polar InGaN/GaN single quantum well structures. This high energy band emerges at carrier densities when the emission from the localized ground states begins to saturate. We attribute this high energy band to recombination involving higher energy less strongly localized electron and hole states that are populated once the localized ground states become saturated; this assignment is supported by the results from an atomistic tight binding model. A particular characteristic of the recombination at the high carrier densities is that the overall forms of the photoluminescence decay curves bear great similarity to those from semiconductor quantum dots. The decay curves consist of plateaus where the photoluminescence intensity is constant with time as a result of Pauli state blocking in the high energy localized states followed by a rapid decrease in intensity once the carrier density is sufficiently low that the states involved are no longer saturated.
\end{abstract}

\section{INTRODUCTION}

Light-emitting diodes with InGaN/GaN quantum well (QW) active regions are widely used in lighting and large area displays. This is because of the high room temperature internal quantum efficiencies achievable by polar InGaN/GaN QW structures. It is widely accepted that the non-radiative effects of the dislocations and point defects are negated due to the localization of carriers at potential minima ${ }^{1-8}$. For polar InGaN/GaN QWs it is predicted $^{6}$ that injected holes will be independently localized at the In concentration fluctuations in the InGaN random alloy whereas electrons would be independently localized by well width fluctuations at the upper well interface. The most obvious indicator of carrier localization is the relatively large low temperature photoluminescence (PL) line width which has been shown to be mainly due to the varying depth of localization of holes ${ }^{6}$. Also, a particularly strong indication of localization is the well-known S-shape temperature dependence of the peak PL energy versus temperature caused by the thermally induced redistribution of the localized carrier(s) that are responsible for the PL line width ${ }^{9-11}$. In the case of InGaN QWs the variation of the energy of the localized hole states, in general, exceeds that of the localized electrons so that the S-shape largely reflects the behavior of the localized holes. Further evidence to support this interpretation has been provided ${ }^{12}$ by the observation of a hole transport limited mobility edge that was revealed in studies of the variation in the recombination spectrum as a function of the excitation energy. This description of separately localized electrons and holes is compatible with the results of low temperature PL time decay measurements ${ }^{13,14}$ where the decay curves are nonexponential.

In general, the observations from measurements and calculations described above have been made at low steady state carrier densities, typically less than $10^{11}$ electron hole pairs $\mathrm{cm}^{-2}$. Over the last few years there have been several reports of the occurrence of a broadening or additional emission band on the high energy side of the emission spectra from InGaN/GaN QW structures as the excitation power densities is increased. A broadening on the high energy side of the PL spectra was observed by Feng et al. ${ }^{15}$ who proposed that it was due to recombination from carriers in weakly localized states and that the associated fast decay component was due to the transfer of carriers from the weakly localized states to strongly localized states. A high energy emission band (HEB) was also observed in high excitation power PL measurements by Sun et al. ${ }^{16}$ which they attributed to recombination involving carriers in extended states. Similar observations in LEDs were reported by Bochkareva et al. and attributed to recombination from weakly localized carriers following the partial filling of the localized tail states ${ }^{17}$. Similarly we have reported ${ }^{18}$ on the emergence at high excitation densities of a rapidly decaying component on the high energy side of the PL spectra that was attributed to weakly localized carriers. In support of this assignment we noted that this observation occurred at higher carrier densities when a reduction in definition of the S-shaped temperature dependence of the PL peak energy was reported by Hammersley et al. and was interpreted as evidence of a saturation of localized states ${ }^{19}$. At room temperature Schulz et al. ${ }^{20}$ also observed a high energy, fast-decaying band, and compared this to 
simulated spectra involving recombination between the ground and first excited electron and hole states. The authors claimed that, for low carrier densities, the quantum confined Stark effect (QCSE) prevents confinement of electron states with sub band indices $n>1$ and the recombination involved electrons where $n=1$ and holes where $n=1$ and $n=2$. However, at high carrier densities the screening of the QCSE enables confinement of electrons with $n=2$ which experience a larger wave function overlap with holes in the $n=2$ sub band resulting in rapidly decaying emission. Importantly, this model neglects the effects of carrier localization as the experiments were performed at room temperature where the authors claim that localization is not important. More recently, Nippert et $a l^{21}$ reported an in-depth study of a similar high energy emission in green emitting InGaN/GaN multiple QW samples which they attributed to recombination involving a confined hole continuum. Of particular note is that the PL decay curves of the high energy band were single exponentials whose time constants decreased with increasing detection energy. This observation was explained as being due to the greater electron/hole wave function overlap of the delocalized holes and electrons. Central to the arguments presented by Schulz et al. ${ }^{20}$ and Nippert et $a l_{.}^{21}$ is that at the high excitation carrier densities localized state saturation occurs resulting in Pauli state blocking similar to that reported in semiconductor quantum dots (QDs) ${ }^{22-26}$. Furthermore, Shahmohammadi et al. ${ }^{27}$ attributed the high energy emission band observed at high excitation densities to recombination involving an electron-hole plasma whose dynamics were dominated by Auger recombination. This conclusion was reached by fitting an ABC model in which A accounts for Shockley-Read-Hall non radiative recombination, $\mathrm{B}$ is the bimolecular radiative decay constant and $\mathrm{C}$ is the Auger constant, to the spectrally integrated PL decay curves.

In this paper, we report on the measurement of low temperature PL spectra and spectrally resolved PL decay dynamics of the emission from a range of InGaN/GaN single QW structures as a function of excited carrier density and compare the results of these measurements with the results of an atomistic model that takes into account ground and excited localized states. In particular, we note the appearance of PL decay curves that are very similar in detail to those reported from semiconductor QDs.

\section{METHODS}

In this section we provide an overview of the methods used in this combined experimental and theoretical investigation of the HEB. We start, in Section II A, with experimental details on growth, structural and optical studies used here. Section II B describes briefly the ingredients of our theoretical framework.

\section{A. Experimental}

Three $c$-plane InGaN/GaN single QW samples were investigated, the In fractions of which were determined using electron energy-loss spectroscopy to be $0.25,0.19$ and 0.15 . The samples were grown by metal-organic chemical-vapor deposition on (0001) sapphire substrates and the QW widths were determined by high-resolution transmission electron microscopy as $3.3 \mathrm{~nm}, 3.2 \mathrm{~nm}$ and $2.9 \mathrm{~nm}$, respectively. Further details regarding the growth and structural analysis of the samples, along with low temperature PL spectroscopy at low excitation carrier densities, are available elsewhere ${ }^{28}$.

Power dependent time integrated PL (TIPL), time resolved PL (TRPL) and PL time-decay measurements were performed using a frequency-tripled mode-locked Ti:sapphire laser with an output photon energy of $4.661 \mathrm{eV}$. The repetition rate of the laser pulses at the sample was adjusted using a pulse picker so that the injected carrier density was able to return to zero before any subsequent pulse was applied. The diameter of the laser spot at the sample surface was measured using a knife edge, and the excited peak carrier densities in the QWs were estimated using the total energy per laser pulse (calculated from the time-averaged power divided by the repetition rate) per unit area. We assumed that that each photon incident on the sample generated one electron-hole pair and all were captured by the QW. The samples were mounted on the cold finger of a closed-cycle He cryostat and held at a temperature of $T=10 \mathrm{~K}$. Light emitted by the sample was dispersed using a $0.75 \mathrm{~m}$ single-grating spectrometer. For TIPL measurements, light was detected using a GaAs photomultiplier tube and processed using standard lock-in amplification techniques. A micro-channel plate (MCP) with a temporal resolution of $\sim 70$ ps was used as the detector for TRPL and PL time-decay measurements. PL decay transients were produced using a time-correlated single-photon counting system.

\section{B. Theoretical Framework}

To study the electronic and optical properties of $c$ plane InGaN QWs on an atomistic level and thus to account for carrier localization effects, we applied the tightbinding (TB) model described in detail in Refs. ${ }^{29,30}$. This model accounts for variations in strain and built-in field arising from random alloy fluctuations on a microscopic level. In accordance with our previous work $^{8}$, we treat InGaN as a random distribution of In and Ga atoms. Additionally, based on the same microstructural analyses and also following previous work ${ }^{6,7}$, well width fluctuations (WWFs) are included in the theoretical modelling and are treated as disk-like structures with a diameter of approximately $5 \mathrm{~nm}$ and a height of 2 monolayers at the upper QW interface. Calculations were performed for QWs with 0.15 and 0.25 In fractions and well widths of 
$3.0 \mathrm{~nm}$ and $3.5 \mathrm{~nm}$, respectively. These values are close to the above reported experimental values.

A central aspect for analyzing the HEB from a theoretical perspective is to study the energy range over which localized states are present in the system. Therefore, not only are the ground states of interest, but also the excited states are of central importance. Additionally, given that the QW region is treated as a random alloy, the calculation of the electronic structure has been repeated several times using different random microscopic configurations so that statistically meaningful results are obtained. Taking all these aspects into account, our calculations of the electronic structure have been performed on 175 different microscopic configurations. For the theoretical study of the QW systems with In fractions of 0.15 and 0.25 we calculated at least 40 electron and 60 hole states per configuration.

Furthermore, to throw light on the HEB, the carrier wave function overlaps play a central role. Following our recent work ${ }^{7}$ we have calculated the modulus wave function overlap (MWFO), $\Omega_{n m}^{\lambda_{1}, \lambda_{2}}=\sum_{i}^{N}\left|\psi_{n, i}^{\lambda_{1}}\right|\left|\psi_{m, i}^{\lambda_{2}}\right|$, between the states $\psi_{n}^{\lambda_{1}}$ and $\psi_{m}^{\lambda_{2}}$. The indices $n, m$ denote the state number while the index $\lambda_{\alpha}$ specifies electron or hole states. For instance if $\lambda_{1}=\lambda_{2}=e, \Omega_{n m}^{e, e}$ describes the MWFO between the different electron states. Also, if $\lambda_{1}=e$ and $\lambda_{2}=h$, the quantity $\Omega_{n m}^{e, h}$ gives the electron and hole MWFO. Using the TB wave functions and since each eigenstate, $\psi_{n}^{\lambda_{1}}$, is connected to a corresponding eigenenergy, the results can be displayed as a function of the energy. To do so and to combine the results from the different microscopic configurations, the overlaps have been grouped together in energy bins of width $30 \mathrm{meV}$ and $20 \mathrm{meV}$ for electrons and holes, respectively. The difference in the bin size accounts for the difference in the localization features of electrons and holes, which takes into account the fact that electrons are less strongly localized when compared to holes. Thus for electrons larger bin sizes can be chosen without masking intrinsic variations in the wave function overlap as a function of energy.

To gain insight into the question of how the wave function overlap changes with carrier density, and thus to shed light onto the carrier density dependence of the HEB, calculations of the energy dependence of $\Omega_{n m}^{\lambda_{1}, \lambda_{2}}$ have been carried out where the carrier occupation at a given energy is described by Fermi-Dirac statistics. Given the strong (hole) localization in the system we assume that the $k$-selection rules are broken similar to the situation in QDs. The calculations were performed as a function of carrier density with the temperature set to be $T=10 \mathrm{~K}$, in accordance with the experiment (see above).

It should be noted that the present calculations have not been performed self-consistently and screening of the electrostatic built-in field is not taken into account. However, we have recently shown in non-polar InGaN/GaN QWs that strong hole localization effects occur even in the complete absence of the built-in field ${ }^{29}$. Furthermore, we have also shown that in these non-polar systems, hole localization occurs over a wide energy range ${ }^{29,31}$. Taking all these factors into account, our TB approach allows us to study the impact of carrier localization effects on the wave function overlap and thus should provide insight into the nature of the HEB.

\section{RESULTS AND DISCUSSION}

The TIPL spectra for the 0.25 In fraction sample as a function of excited peak carrier density are shown in Fig. 1. At the lowest carrier density (Fig. 1(a)) the peak energy of the strongest feature is at $2.135 \mathrm{eV}$ and we assign this to recombination involving electrons and holes localized at a range of the lowest localized energy states (i.e. the ground states). The peaks at $2.045 \mathrm{eV}$ and $1.953 \mathrm{eV}$ are the first and second longitudinal optical phonon replicas ${ }^{28}$ of the ground state recombination, respectively. As the excitation density is increased, a broadening on the high energy side of the spectrum occurs (Fig. 1(b)). As the carrier density is increased further, a distinct HEB emerges (Figs. 1(c) and 1(d)). At the highest excited carrier density the HEB extends to almost $400 \mathrm{meV}$ above the low excitation density peak emission energy. The intensity oscillations present on the HEB are caused by Fabry-Perot interference effects ${ }^{28}$.

Numerous broad luminescence bands have been identified in bulk GaN associated with various defects and impurities $^{32}$. In order to rule out the assignment of the HEB to one of these bands, power dependent TIPL was also performed on the 0.15 and 0.19 In fraction samples and the normalized TIPL spectra for all three samples at an excited carrier density of $2.5 \times 10^{13} \mathrm{~cm}^{-2}$ per pulse are shown in Fig. 2. The HEB occurs exclusively on the high energy side of the main emission peak for each sample. This means that we can rule out defect luminescence from the bulk GaN as the source of the HEB.

At this point in our discussion we draw upon the results of the theoretical model described earlier. Fig. 3 (a) shows the energy resolved MWFO $\Omega^{e e}$ in the QW with 0.25 In fraction for low $\left(5.0 \times 10^{12} \mathrm{~cm}^{-2}\right)$, medium $\left(1.5 \times 10^{13} \mathrm{~cm}^{-2}\right)$ and high $\left(2.5 \times 10^{13} \mathrm{~cm}^{-2}\right)$ carrier densities. For all these calculations, the zero of the energy scale is the unstrained GaN valence band edge. The dark areas indicate that the wave function overlap in the corresponding energy interval is basically zero whilst the white regions reflect an overlap close to one. Please note that, given that the wave functions are normalized, $\Omega^{e e}$ cannot exceed a value of one. Starting with the high carrier density results (right column in Fig. 3(a)), we find a very high overlap between the electron states in the different energy ranges. When reducing the carrier density and given that the calculations have been performed at low temperatures $(T=10 \mathrm{~K})$, the carriers populate only a subset of the available electron states. This leads to the appearance of the black area above an energy of approximately $3.15 \mathrm{eV}$. This becomes even more extreme for 


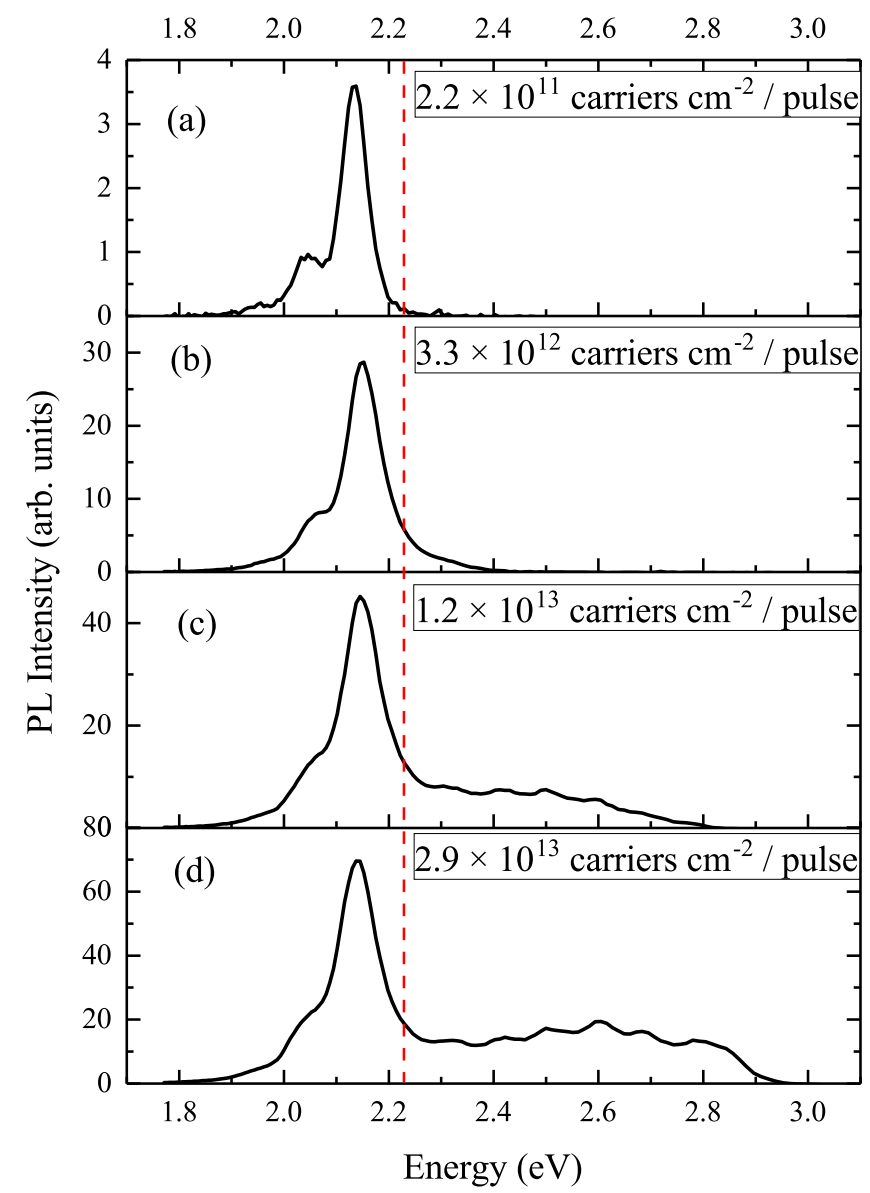

FIG. 1: Carrier density dependent TIPL of the $0.25 \mathrm{In}$ fraction sample. The dashed red line indicates the demarcation between the ground state recombination and the HEB used for acquiring the data plotted in Fig. 8.

the lowest carrier density. For the lowest carrier density and at $T=10 \mathrm{~K}$ only electron states at the "conduction band" edge (bottom left of each sub figure in Fig. 3(c)) are populated.

The same analysis has been carried for the hole states. Figure 3(b) displays $\Omega^{h h}$ at the same carrier densities and with the same color coding as in Fig. 3(a) for the electrons. When looking at the high carrier density regime (right column in Fig. 3(b)), for the hole states we find a vastly different behavior compared to the electrons (right column in Fig. 3(a)). While for the electrons across the full energy range very high wave functions overlaps are observed, for the holes $\Omega^{h h}$ varies strongly with energy. Near the "valence band" edge, which corresponds to the bottom left corner of Fig. 3(b) (energies between approximately $0.9 \mathrm{eV}$ and $1 \mathrm{eV}$ ), we find a region of strongly localized states with very little overlap between each other. This region of strongly localized states is followed by a region of "semi-localized states" (energies between approximately $0.7 \mathrm{eV}$ and $0.9 \mathrm{eV}$ ) where the wave function overlap between the different hole states is greater when

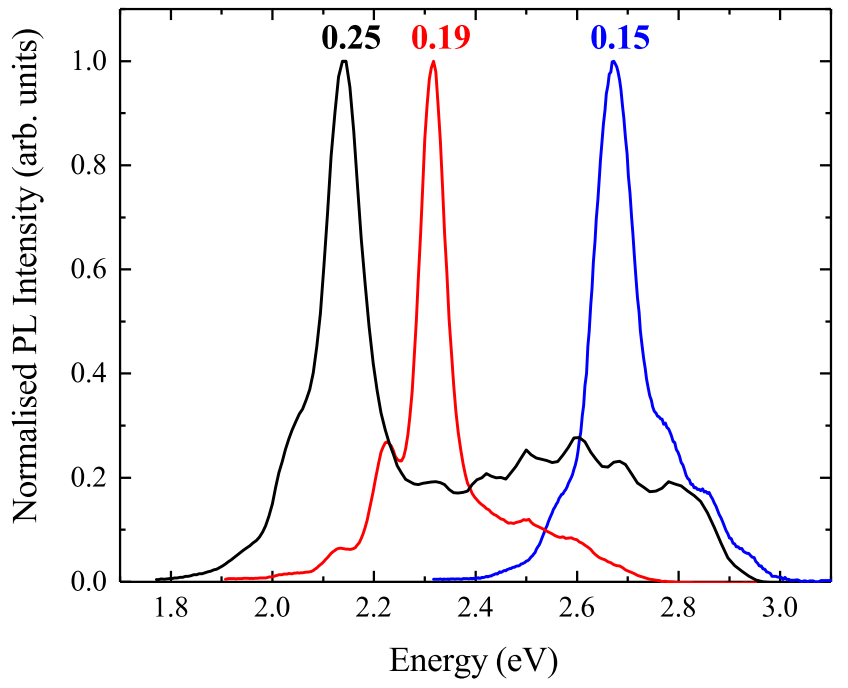

FIG. 2: TIPL spectra performed at carrier density of $2.5 \times 10^{13} \mathrm{~cm}^{-2}$ per pulse for the QWs with In fractions $0.25,0.19$ and 0.15 .

compared to the strongly localized region. Finally, $\Omega^{h h}$ further increases in the upper right corner for the higher energy hole states. In this high energy region the holes are essentially delocalized. From this analysis we conclude that with increasing carrier density the highest occupied hole states become progressively delocalized with increasing carrier concentration. This finding underpins our previous conclusion ${ }^{6,30}$ that at low carrier densities the PL spectrum is dominated by the variation in energy of strongly localized hole states.

These results beg the question of how the electron hole wave function overlap changes with increasing carrier density. Figure 3(c) depicts $\Omega^{\text {eh }}$ using the same carrier densities and color coding as before. We start our analysis here with the lowest carrier density. In what follows, it is important to keep in mind the results of the calculations for the electron-electron and hole-hole wave function overlaps and that electrons and holes are localized at opposite sides of the QW due to the presence of the macroscopic built-in field along the growth direction. Therefore, taking also the strong localization effects for holes into account, the electron and hole wave function overlap is very small compared to the electron-electron or hole-hole wave function overlaps. Thus at low carrier densities and low temperatures the PL spectrum mainly involves the strongly localized hole states. Increasing the carrier density now results in the situation where holes start to populate semi-localized and eventually delocalized states. For the electrons, as discussed above, the localization effects at higher carrier densities are of secondary importance when compared to the holes. Thus, in the case of the highest carrier density (right column of Fig. 3(c)), we find a strong increase in the electron-hole wave function overlap. Again, this is introduced by the 
fact that the delocalized states are populated and that the strongly localized hole states are saturated. In general this picture is consistent with the experimental findings and conclusions that the PL spectrum at low carrier densities is dominated by localized states, and the HEB occurs after these states have been saturated and extra recombination "channels" appear on the high energy side of the low carrier density PL peak. From this analysis we would also expect faster (radiative) recombination lifetimes for the HEB. While it is tempting to use this explanation for the form of the experimental spectra, it is worth drawing attention at this point to the potential involvement of non-radiative recombination centers. While at low carrier densities carrier localization can prevent the carriers from recombining non-radiatively, at higher carrier densities this may no longer be the case as we have shown above. We will return to this question later in our discussion.

On the experimental side, the nature of the HEB was investigated in greater depth using TRPL spectroscopy. The results of these studies for the 0.25 In fraction sample are displayed in Fig. 4. These spectra show that the PL intensity of the HEB decays rapidly on a time scale of nanoseconds. The latest time window shown (29.9$30.2 \mathrm{~ns}$, ) gives a spectrum that closely resembles the low excited carrier density spectrum in Fig. 1. These observations are consistent with the theoretical work discussed above whereby we would expect the HEB, which involves the weakly localized carriers, to recombine radiatively on a much faster time scale than the low density PL peak.

The TIPL and TRPL spectra shown in Figs. 1 and 4, respectively, are similar to those reported by Sun et al. ${ }^{16}$ and Nippert et $a .^{21}$. In order to investigate further the temporal behavior of the HEB, PL decay transients were measured at various detection energies across the spectrum at a peak excited carrier density of $2.2 \times 10^{13} \mathrm{~cm}^{-2}$ per pulse and are shown in Fig. 5. At the highest detection energies $(2.883 \mathrm{eV}$ and $2.725 \mathrm{eV})$ the decays are exponential; the peaks in the decays at $1.7 \mathrm{~ns}$ are associated with the instrumental response. As the detection energy is decreased the final parts of the decays are exponential but these components are delayed in time by plateau regions which increase in duration with decreasing detection energy.

We now consider in detail the form of the PL decay curves shown in Fig. 5, in particular the plateau regions. This type of behavior has been reported previously ${ }^{23-26}$ in high excitation density studies of the recombination dynamics of a variety of semiconductor QD systems. In general, in these reports, as the excitation density was increased recombination involving excited states emerged on the high energy side of the ground state recombination. As we believe that the recombination in the InGaN QWs involves localized carriers then we draw a parallel between the system we have studied in this paper and semiconductor QDs. Within this framework we have used the rate equation model described by Grosse et al. ${ }^{26}$ to shed further light on the nature of the recombination in
InGaN QWs. Based on the model of Grosse et al. ${ }^{26}$ we describe the dynamics of the localized hole states as follows:

$$
\frac{d N_{i}(t)}{d t}=-\frac{N_{i}(t)}{\tau_{\mathrm{rec}, i}}+\frac{N_{i+1}(t)}{\tau_{\mathrm{rel}, i+1}} f_{i}-\frac{N_{i}(t)}{\tau_{\mathrm{rel}, i}} f_{i-1} .
$$

Here Pauli-blocking (state filling) effects are described by the factor $f_{i}$ in the above rate equation model which means that carriers can only relax to non-occupied (localized) states. The state filling factor, $f_{i}$, is defined by $f_{i}=\left[D_{i}-N_{i}(t)\right] / D_{i}$, where $D_{i}=2 i$ accounts for (spin) degeneracies of the localized states in the (quantum dot) model. The time dependence of the (mean) carrier population in level $i$ is denoted by $N_{i}(t)$. This model requires as input the recombination (either radiative or non-radiative) lifetimes, $\tau_{\mathrm{rec}, i}$, for the different levels, acoustic phonon and Coulomb scattering times, which are both combined in the relaxation time, $\tau_{\text {rel }, i}$. Following Ref. ${ }^{21}$, we use a Coulomb scattering time of $1 \mathrm{ps}$. We apply for the lifetimes, $\tau_{\mathrm{rec}, i}$, those values for the exponential portions of the decays shown in Fig. 5. For the phonon scattering times we rely on the values from many-body calculations of InGaN-based $\mathrm{QDs}^{33}$, where values of 10 ps have been reported for the phonon scattering times involving holes. Using this approach, we have modelled the five PL transients displayed in Fig. 5 for recombination energies between $2.234 \mathrm{eV}$ and $2.583 \mathrm{eV}$. The results of the rate equation model using this QD-like description are shown in Fig. 6. In this figure "Ground state" $\left(N_{1}\right)$ denotes the lowest energy level, which following Fig. 5 corresponds to the data with energy $2.234 \mathrm{eV}$. Conversely, the "Third excited states" $\left(N_{4}\right)$ should be compared to the spectrum with a detection energy of $2.583 \mathrm{eV}$. It should be stressed here that a one-to-one comparison between theory and experiment is not the prime focus, but Fig. 6 reveals that this simple rate equation model indeed captures the main features of the experimental spectra. More specifically, the behavior in Fig. 6 is reflected in the PL decay curves shown in Fig. 5 where at high excitation densities and at early times regions of relatively constant PL intensity were observed, i.e. forming a plateau, which is due to states being refilled by carriers from higher lying states. We attribute the plateau regions observed on the decays measured at the detection energies of $2.234 \mathrm{eV}, 2.339 \mathrm{eV}, 2.455 \mathrm{eV}$, and $2.583 \mathrm{eV}$ to this type of behavior. Thus the time decay measurements, in combination with our theoretical results, lend weight to our proposal that the HEB is due to recombination of carriers occupying semi-localized excited states. Further evidence for this is provided by PL time decay measurements performed on the $0.25 \mathrm{In}$ fraction sample at two different excited carrier densities and at detection energy $2.362 \mathrm{eV}$, shown in Fig. 7. As the excited carrier density is increased, the duration of the plateau region of the decay increases while the time constant, $\tau$, of the final exponential part of the decay remains constant with a characteristic time constant of 

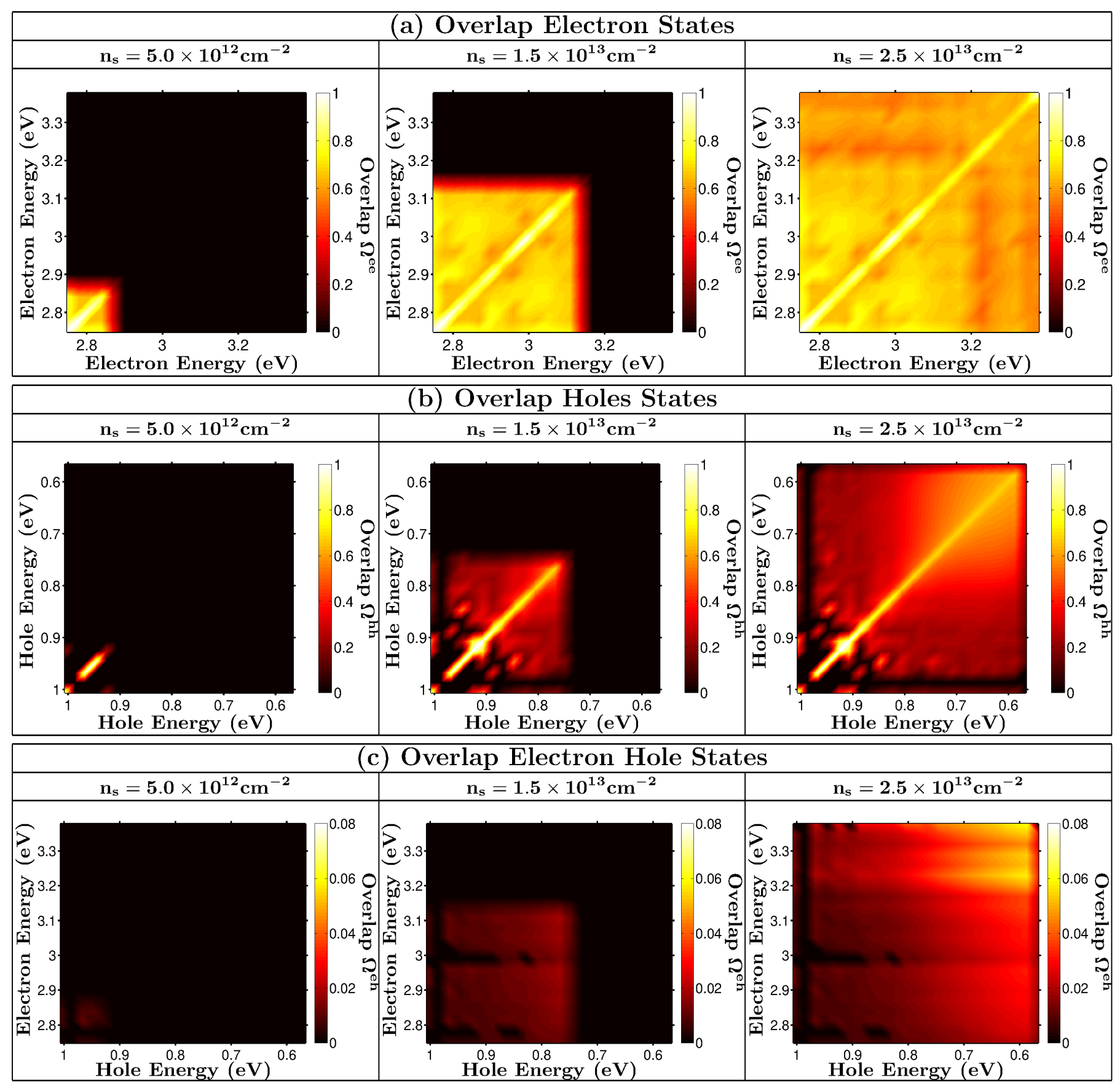

FIG. 3: (a) Energy resolved modulus electron-electron wave function overlap, $\Omega^{e e}$, for sheet carrier densities, $n_{s}$, of $n_{s}=5.0 \times 10^{12} \mathrm{~cm}^{-2}$ (left), $n_{s}=1.5 \times 10^{13} \mathrm{~cm}^{-2}$ (middle) and $n_{s}=2.5 \times 10^{13} \mathrm{~cm}^{-2}$, (b) Energy resolved modulus hole-hole wave function overlap $\Omega^{h h}$ for sheet carrier densities of $n_{s}=5.0 \times 10^{12} \mathrm{~cm}^{-2}$ (left), $n_{s}=1.5 \times 10^{13} \mathrm{~cm}^{-2}$ (middle) and $n_{s}=2.5 \times 10^{13} \mathrm{~cm}^{-2}$, (c) Energy resolved modulus electron-hole wave function overlap $\Omega^{\text {eh }}$ for sheet carrier densities of $n_{s}=5.0 \times 10^{12} \mathrm{~cm}^{-2}$ (left), $n_{s}=1.5 \times 10^{13} \mathrm{~cm}^{-2}$ (middle) and $n_{s}=2.5 \times 10^{13} \mathrm{~cm}^{-2}$. The calculations have been performed for a $\operatorname{In}_{0.25} \mathrm{Ga}_{0.75} \mathrm{~N} / \mathrm{GaN}$ quantum well of width $3.5 \mathrm{~nm}$. The zero of the energy scale is the unstrained $\mathrm{GaN}$ valence band edge.

2.0 ns. This again is compatible with the QD studies ${ }^{26}$, whereby increasing the excited carrier density leads to occupation of even higher energy states resulting in the refilling lasting longer.
Next, we return to a more detailed consideration of the factors responsible for the form of the decay curves after the plateau regions. In these time regimes the (semi)localized states are not subject to being refilled from 


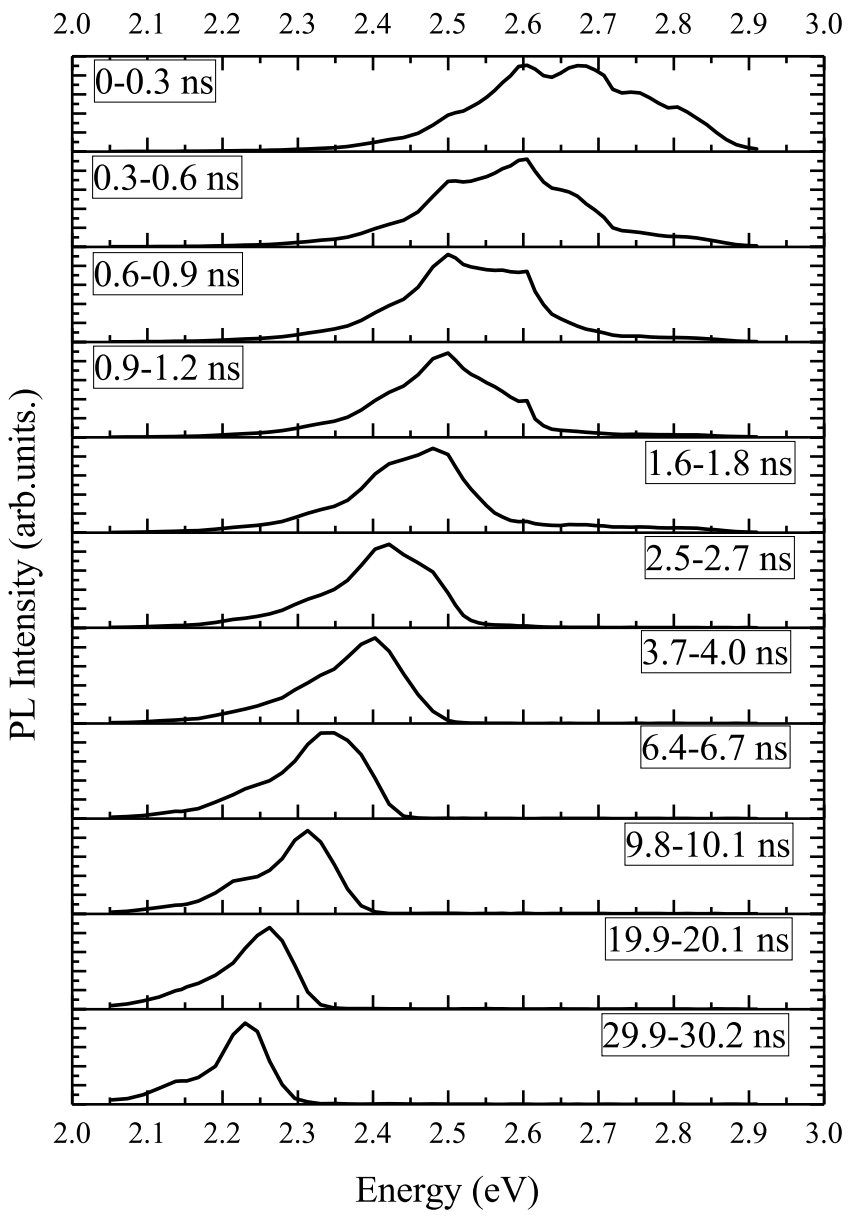

FIG. 4: Time resolved PL spectra for the $0.25 \mathrm{In}$ fraction sample with spectra for each time window shown separately on an arbitrary intensity scale.

higher lying states and the carriers can recombine nonradiatively or radiatively. In the rate equation model described above we were not specific about the nature of the recombination process(es) as this is not a necessary aspect of this approach. In general the forms of the decays are exponential, which is radically different from those of the ground states that decay non-exponentially ${ }^{13,14}$, reflecting the recombination of spatially independently localized electrons and holes. For the case of radiative recombination across the HEB we may anticipate, for the recombination of carriers occupying excited states, that the radiative decay would occur not only on a faster time scale than the ground state originating from the larger electron-hole wave function overlaps but also that the radiative decay rate would increase with increasing detection energy across the HEB, again reflecting the greater electron-hole wave function overlap predicted by the results of our TB model shown in Fig. 3. However, when we observe the excited state emission, the quantum efficiency of the integrated PL intensity is less than 1, i.e. efficiency droop occurs. Thus it is tempting to conclude that the decay constants in the non-plateau regions

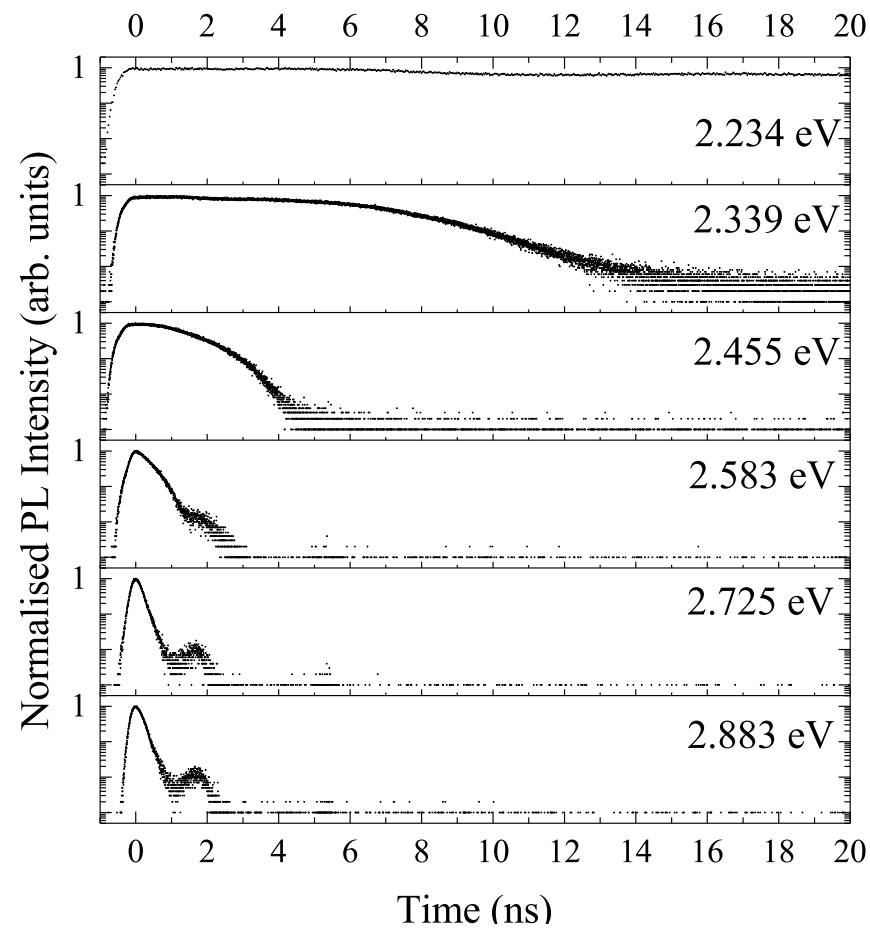

FIG. 5: PL decay transients for a peak excited carrier density of $2.2 \times 10^{13} \mathrm{~cm}^{-2}$ per pulse for a range of detection energies for the 0.25 In fraction sample.

across the HEB are influenced by non-radiative processes possibly because, as discussed above, the carrier localization is weaker. To test this hypothesis we plot in Fig. 8 the integrated intensities of the emission involving mainly localized carriers (ground state emission) and the HEB as a function the excited carrier density. Separating the two emission components is somewhat difficult, especially at the low injection level when the HEB is a shoulder on the side of the ground state emission; the dividing line between the two emission bands is the red dashed line in Fig. 1. Also great care must be taken in the interpretation of this data as the values on the carrier density axis cannot be applied independently to the two emission bands as the plot itself implies. This stems from the situation that the estimated carrier densities marked on the axis are in fact "split" between the two recombination channels. Nevertheless, useful information can be extracted from Fig. 8. First we turn our attention to the ground state emission (black squares). For carrier densities above $5 \times 10^{12} \mathrm{~cm}^{-2}$ per pulse, the integrated intensity clearly increases sub linearly. This is not due to any non-radiative process as that would be apparent in the decay data but rather it involves saturation of the localized state(s) involved in the ground state recombination. Now we turn our attention to the HEB (red circles) where, taking into account the caveat on interpreting the data in Fig. 8, the integrated intensity of the HEB increases almost linearly with increasing excitation density. This suggests, bearing in mind the reservations 


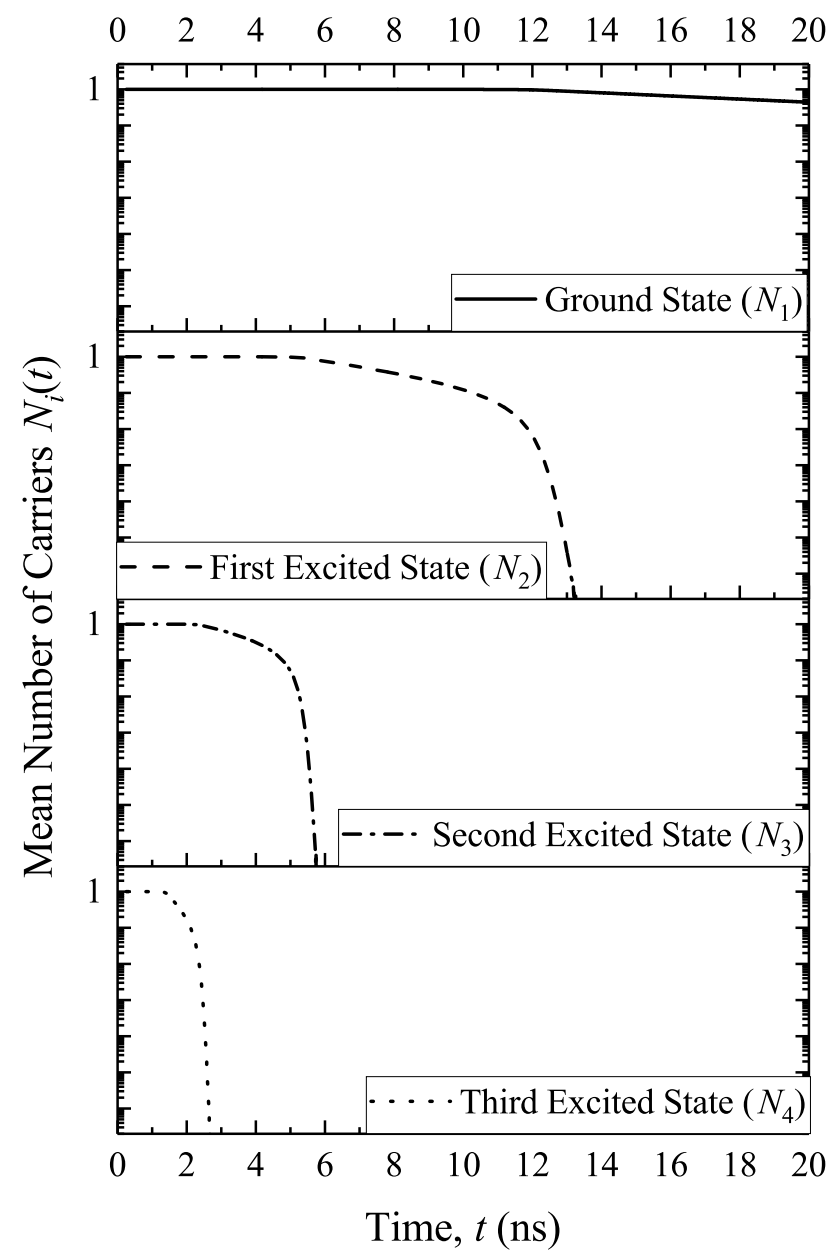

FIG. 6: Temporal evolution of the mean number of carriers (holes), $N_{i}(t)$, occupying a quantum dot like model system with four localized states. The lowest energy state is denoted by "Ground State $\left(N_{1}\right)$ "; the energetically highest state is denoted by "Third Excited State $\left(N_{4}\right)$ ". Details of the rate equation model used here are given in the main text.

expressed above, that the decay transients from the HEB are dominated by radiative processes. It is worth noting that this conclusion is different from that reached in the work by Shahmohammadi et al. ${ }^{27}$, where it was reported that a fast transient was observed on the decay of the spectrally integrated PL at high carrier densities that was attributed to Auger recombination. We do not observe such behavior as our experiments were carried out in a significantly different way in that we measure the spectrally resolved PL decay transients as opposed to spectrally integrated measurements.

One further interesting observation made here relates to the form of the radiative decay transients. As briefly discussed above, experimentally we observe singleexponential decay transients when studying the HEB. This finding is in strong contrast to the form of the decay transients of the ground states which are non-

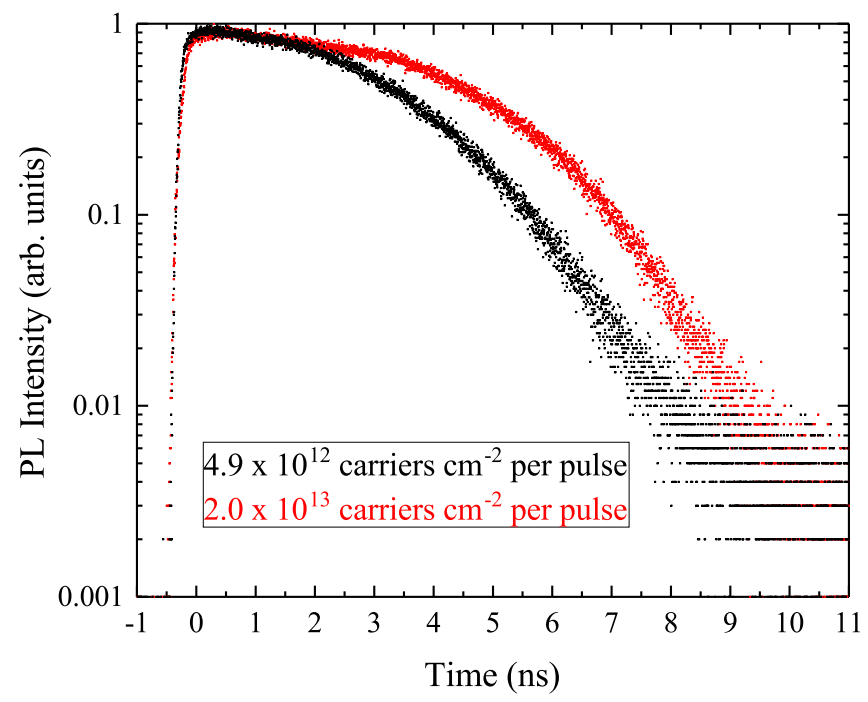

FIG. 7: PL time decay transients for the different peak excited carrier densities indicated at a detection energy of $2.362 \mathrm{eV}$.

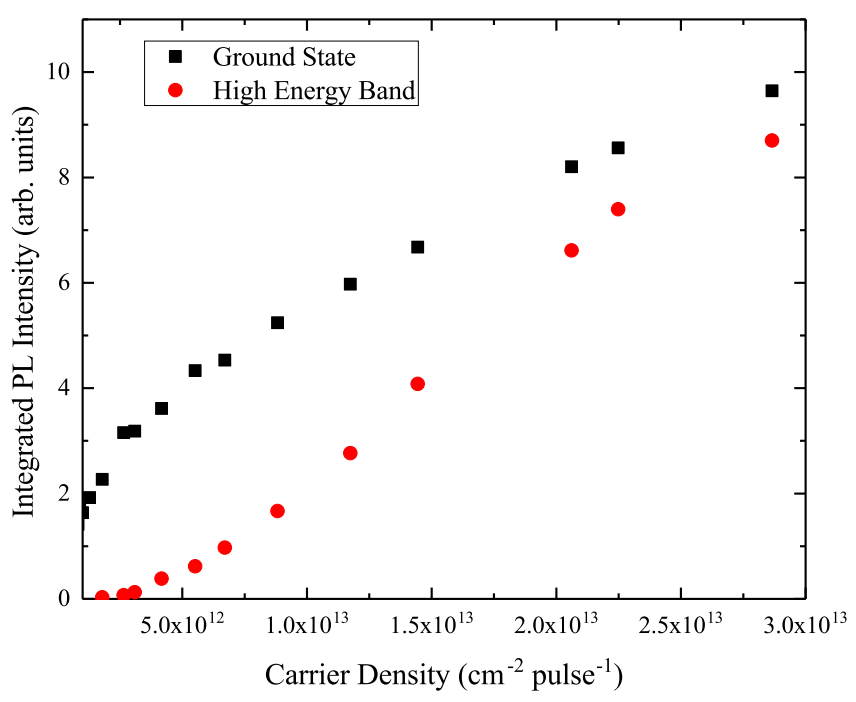

FIG. 8: Integrated PL intensity of the ground state recombination and the high energy band as a function of the total excited peak carrier density.

exponential. On a first glance, the results of the TB model might suggest that at any one recombination energy there will be a range of values for the overlap of electron and hole wave functions. Based on this observation, non-exponential decay curves could be expected. However, it is important to point out that Coulomb effects have not been taken into account in our theoretical study here. While our approach is sufficient to address our main objective here, namely to gain insight into the energy distribution of localized states and how carrier localization features change with energy and carrier density, Coulomb effects are obviously central when analyzing recombination dynamics, especially when deal- 
ing with weakly localized carriers that contribute to the HEB. It is beyond the scope of this work to study carrier and recombination dynamics. Nonetheless, the changes in the decay transients provide a clear starting point for future investigations since understanding this feature in detail will help to shed further light onto the fundamental (radiative) recombination properties of $\mathrm{InGaN} / \mathrm{GaN}$ QW systems.

\section{SUMMARY}

In summary, we have observed the appearance of a HEB in the PL spectra of a series of InGaN single QW structures. Comparisons of the spectra with the results of a TB model which accounts for variations in strain and built-in field arising from alloy fluctuations on a microscopic level lead us to conclude that the HEB arises from carriers confined in weakly localized states. This conclusion is supported by the results of detailed time decay measurements taken across the spectra which strikingly exhibit plateaus similar to those observed in semiconductor QDs. As illustrated by a rate equation model, these plateaus are due to Pauli or state blocking effects associ- ated with the zero dimensional character of the localized states. Once the carrier densities have reduced such that the state blocking no longer occurs, the PL decreases in time, at a rate dictated by radiative processes. The overall spectral dependence of the radiative recombination, whereby the rates increase with increasing detection energy, is compatible with the results of our TB model. This model also reveals that the states contributing to the lower energy emission involve the more strongly localized states where the electron-hole wave function overlap gets progressively weaker as the recombination rate decreases.

\section{ACKNOWLEDGMENTS}

This work was carried out with the support of the United Kingdom Engineering and Physical Sciences Research Council under grant Nos. EP/I012591/1, $\mathrm{EP} / \mathrm{M} 010627 / 1$ and EP/M010589/1. SS acknowledges support from the Science Foundation Ireland under grant Nos. $13 /$ SIRG/2210 and 17/CDA/4789. Additional research data supporting this publication are available from the University of Manchester repository at http: //dx.doi.org/10.17632/w4fz4d8mfz.1.
* Contact email: george.christian@manchester.ac.uk

1 S. Chichibu, K. Wada, and S. Nakamura, Applied Physics Letters 71, 2346 (1997).

2 K. L. Teo, J. S. Colton, P. Y. Yu, E. R. Weber, M. F. Li, W. Liu, K. Uchida, H. Tokunaga, N. Akutsu, and K. Matsumoto, Applied Physics Letters 73, 1697 (1998).

3 J. Bai, T. Wang, and S. Sakai, Journal of Applied Physics 88, 4729 (2000).

${ }^{4}$ I. L. Krestnikov, N. N. Ledentsov, A. Hoffmann, D. Bimberg, A. V. Sakharov, W. V. Lundin, A. F. Tsatsul'nikov, A. S. Usikov, Z. I. Alferov, Y. G. Musikhin, and D. Gerthsen, Physical Review B 66, 155310 (2002).

${ }^{5}$ H. Schömig, S. Halm, A. Forchel, G. Bacher, J. Off, and F. Scholz, Physical Review Letters 92, 106802 (2004).

6 D. Watson-Parris, M. J. Godfrey, P. Dawson, R. A. Oliver, M. J. Galtrey, M. J. Kappers, and C. J. Humphreys, Physical Review B 83, 115321 (2011).

7 D. S. P. Tanner, M. A. Caro, E. P. O'Reilly, and S. Schulz, RSC Advances 6, 64513 (2016).

8 M. J. Galtrey, R. A. Oliver, M. J. Kappers, C. J. Humphreys, P. H. Clifton, D. Larson, D. W. Saxey, and A. Cerezo, Journal of Applied Physics 104, 013524 (2008).

9 P. G. Eliseev, P. Perlin, J. Lee, and M. Osinski, Applied Physics Letters 71, 569 (1997).

10 Y.-H. Cho, G. H. Gainer, a. J. Fischer, J. J. Song, S. Keller, U. K. Mishra, and S. P. DenBaars, Applied Physics Letters 73, 1370 (1998).

11 O. Rubel, M. Galluppi, S. D. Baranovskii, K. Volz, L. Geelhaar, H. Riechert, P. Thomas, and W. Stolz, Journal of Applied Physics 98, 063518 (2005).

12 W. E. Blenkhorn, S. Schulz, D. S. P. Tanner, R. A. Oliver, M. J. Kappers, C. J. Humphreys, and P. Dawson, Journal of Physics: Condensed Matter 30, 175303 (2018).

13 J. A. Davidson, P. Dawson, T. Wang, T. Sugahara, J. W. Orton, and S. Sakai, Semiconductor Science and Technology 15, 497 (2000).

14 A. Morel, P. Lefebvre, S. Kalliakos, T. Taliercio, T. Bretagnon, and B. Gil, Physical Review B 68, 045331 (2003).

15 S.-W. Feng, Y.-C. Cheng, Y.-Y. Chung, C. C. Yang, M.-H. Mao, Y.-S. Lin, K.-J. Ma, and J.-I. Chyi, Applied Physics Letters 80, 4375 (2002).

16 G. Sun, G. Xu, Y. J. Ding, H. Zhao, G. Liu, J. Zhang, and N. Tansu, Applied Physics Letters 99, 081104 (2011).

17 N. I. Bochkareva, Y. T. Rebane, and Y. G. Shreter, Applied Physics Letters 103, 191101 (2013).

18 M. J. Davies, T. J. Badcock, P. Dawson, M. J. Kappers, R. A. Oliver, and C. J. Humphreys, Applied Physics Letters 102, 022106 (2013).

19 S. Hammersley, D. Watson-Parris, P. Dawson, M. J. Godfrey, T. J. Badcock, M. J. Kappers, C. McAleese, R. A. Oliver, and C. J. Humphreys, Journal of Applied Physics 111, 083512 (2012).

20 T. Schulz, A. Nirschl, P. Drechsel, F. Nippert, T. Markurt, M. Albrecht, and A. Hoffmann, Applied Physics Letters 105, 181109 (2014).

${ }^{21}$ F. Nippert, A. Nirschl, T. Schulz, G. Callsen, I. Pietzonka, S. Westerkamp, T. Kure, C. Nenstiel, M. Strassburg, M. Albrecht, and A. Hoffmann, Journal of Applied Physics 119, 215707 (2016).

22 M. J. Steer, D. J. Mowbray, W. R. Tribe, M. S. Skolnick, M. D. Sturge, M. Hopkinson, A. G. Cullis, C. R. Whitehouse, and R. Murray, Physical Review B 54, 17738 (1996). 
${ }^{23}$ F. Adler, M. Geiger, A. Bauknecht, F. Scholz, H. Schweizer, M. H. Pilkuhn, B. Ohnesorge, and A. Forchel, Journal of Applied Physics 80, 4019 (1996).

24 M. Grundmann and D. Bimberg, Physical Review B 55, 9740 (1997).

${ }^{25}$ P. D. Buckle, P. Dawson, S. A. Hall, X. Chen, M. J. Steer, D. J. Mowbray, M. S. Skolnick, and M. Hopkinson, Journal of Applied Physics 86, 2555 (1999).

26 S. Grosse, J. H. H. Sandmann, G. von Plessen, J. Feldmann, H. Lipsanen, M. Sopanen, J. Tulkki, and J. Ahopelto, Physical Review B 55, 4473 (1997).

27 M. Shahmohammadi, W. Liu, G. Rossbach, L. Lahourcade, A. Dussaigne, C. Bougerol, R. Butté, N. Grandjean, B. Deveaud, and G. Jacopin, Physical Review B 95, 125314 (2017).

${ }^{28}$ D. M. Graham, A. Soltani-Vala, P. Dawson, M. J. Godfrey, T. M. Smeeton, J. S. Barnard, M. J. Kappers, C. J.
Humphreys, and E. J. Thrush, Journal of Applied Physics 97, 103508 (2005).

29 S. Schulz, D. S. P. Tanner, E. P. O'Reilly, M. A. Caro, F. Tang, J. T. Griffiths, F. Oehler, M. J. Kappers, R. A. Oliver, C. J. Humphreys, D. Sutherland, M. J. Davies, and P. Dawson, Applied Physics Letters 109, 223102 (2016).

30 S. Schulz, M. A. Caro, C. Coughlan, and E. P. O'Reilly, Physical Review B 91, 035439 (2015), arXiv:1501.05482.

31 D. P. Tanner, M. A. Caro, E. P. O'Reilly, and S. Schulz, physica status solidi (b) 253, 853 (2016).

32 M. A. Reshchikov and H. Morkoc, Journal of Applied Physics 97, 061301 (2005).

33 T. R. Nielsen, P. Gartner, and F. Jahnke, Physical Review B 69, 235314 (2004). 